\title{
Avances en la formación post residencia. La creación de un marco básico común de referencia
}

\author{
Carlos Tello, Santiago Vedoya, Carlos Autorino, Rodrigo Remondino, José Varaona, Carlos Pelaez, \\ Julián Romagnoli, Roberto Valentini, Juan Pablo Torrano, Federico Sartor, Fernando Catapano, \\ Mariano Parisi, Alejandro Fazio, Silvia Reciniello, Carlos Sancineto, Lic. Fabiana Reboiras, Lic. Clara Facioni \\ Comité de Residencias y Sistemas Equivalentes, Asociación Argentina de Ortopedia y Traumatología, Buenos Aires, Argentina
}

\section{INTRODUCCIÓN}

De manera creciente, la educación y formación de los especialistas, después de haber completado la residencia, se continúan en espacios asistenciales para profundizar el entrenamiento en disciplinas más específicas de la Ortopedia y Traumatología. Este fenómeno se produce también en otras disciplinas, como cirugía general, urología, neurocirugía, entre otras. Si bien la Ortopedia y Traumatología no está considerada como materia básica para estamentos administrativos sanitarios, desde la óptica funcional lo es. Luego de la graduación, en la Argentina, parte de los médicos deciden continuar con una formación de posgrado, al especializarse en alguna rama de la asistencia en salud. En el caso de la Ortopedia y Traumatología, se dedican cuatro años de formación como especialista en sedes acreditadas. Al finalizar y especialmente en zonas urbanas de gran complejidad y desarrollo, necesitan profundizar su preparación en subespecialidades. El desarrollo permanente, el incremento de los conocimientos, la responsabilidad profesional y legal, la demanda de los prestadores de salud y la misma población lo requieren. El crecimiento de las subespecialidades y su desarrollo han llevado a la creación de unidades y hasta servicios de dedicación exclusiva a distintos aspectos de la Ortopedia. Inclusive se han enriquecido con la incorporación de otras disciplinas médicas; son ejemplos la especialidad espinal o de columna vertebral que ha incorporado neurocirujanos y la de mano que se ha nutrido con cirujanos plásticos.

En la actualidad, muchos Servicios de Ortopedia y Traumatología, acompañados por el desarrollo de la formación en subespecialidad, han conformado sectores y equipos específicos: Cadera y rodilla, Columna, Miembro Superior y Mano, Tobillo y pie, Ortopedia Infantil, Oncología, Neuroortopedia, Trauma, Deporte, Artroscopia y seguramente vendrán más. En algunas instituciones, determinadas subespecialidades de la Ortopedia ya se han constituido como Servicios. En estos espacios, sectores o equipos es donde concurren los médicos egresados de la Residencia de Traumatología y Ortopedia para proseguir especializando sus conocimientos y competencias. Esta situación, nada nueva, ha sido muy fructífera para el conocimiento y elevación de los estándares de calidad médica tanto asistencial como académica. Con el tiempo se ha observado la necesidad de consensuar algunos aspectos comunes, como ser: la denominación, el tiempo y el programa. Se encuentran variadas coincidencias como también una gran dispersión entre las diferentes ofertas de formación en la post residencia. Es claro que cada una de ellas puede tener distinto tiempo de formación y dedicación, pero a este aspecto, como a otros, se los prefiere ubicar dentro de un marco común que procure aspectos básicos en el que coincidan.

En 2014, el Comité de Residencias y Sistemas Equivalentes de la Asociación Argentina de Ortopedia y Traumatología obtuvo la modificación estatutaria de la Asociación en el sentido de ocuparse de los sistemas de especialización en Ortopedia y Traumatología, y aquellos que se hallen involucrados en el posgrado. Las funciones del Comité están dirigidas al control, la evaluación, la acreditación y el apoyo de los sistemas educativos para los médicos que buscan su especialización básica y post-básica en forma de Residencias, Concurrencias, Becarios, Pasantes u otra denominación que se tenga o tuviere para las formaciones de especialistas como de subespecialidades.

Dr. CARLOS TELLO • tello@fibertel.com.ar ID

Cómo citar este artículo: Tello C, Vedoya S, Autorino C, Remondino R, Varaona J, Pelaez C, et al. Avances en la formación post residencia. La creación de un marco básico común de referencia. Rev Asoc Argent Ortop Traumatol 2019;84(2):178-187. http://dx.doi.org/10.15417/issn.1852-7434.2019.84.2.976 
Por ello es que, desde esos momentos, una de las líneas de trabajo fue la de dedicarse al estudio de situaciones y elaborar una táctica para lograr, en forma consensuada con las distintas Asociaciones y Sociedades, los criterios mínimos y las recomendaciones para la formación post residencia. El objetivo de este trabajo es presentar el marco de referencia para dicha formación.

\section{MÉTODO}

En los últimos cuatro años, se realizaron encuentros inicialmente, en forma grupal, con los directivos de las Asociaciones y Sociedades de la especialidad, luego en forma individual, con los representantes de estas. Es, durante 2018, que se convoca a expertas, licenciadas en Educación que aportaron sus perspectivas y diseñaron una metodología de encuentros para discutir y definir consensuadamente un documento preliminar básico que sirviera como marco de referencia para la educación post residencia. Se convocó a todas las Asociaciones y Sociedades a que enviaran sus representantes. Se efectuaron cuatro reuniones, de dos horas de duración, en las que el eje central fue la participación de los expertos en las diferentes subespecialidades y el trabajo grupal. La modalidad de trabajo, durante las tres primeras y luego de una introducción conceptual, fue que los distintos representantes, junto con miembros del Comité de Residencias se dividieran en grupos y discutieran el temario propuesto para cada reunión. Al finalizar cada sesión, se presentaban las conclusiones. En la última reunión, se dio forma y se redactó el documento final (Anexo).

\section{DISCUSIÓN}

Desde 2007, la American Academy of Orthopaedic Surgeons (AAOS) y la American Orthopedic Association (AOA) han tratado de promocionar un proceso coordinado para el entrenamiento post residencia o fellowship. ${ }^{1} \mathrm{El}$ 90\% de los residentes de Ortopedia prosiguen su entrenamiento como fellows, según lo que afirman varios autores en los EE.UU. ${ }^{2}$ Evidentemente la educación médica se ha prolongado en el tiempo. La Residencia se halla estructurada y aceptada con parejas definiciones, marcos de enseñanza y evaluación ya aquilatados. Con menor o mayor variación se desarrollan según la complejidad y necesidad de los diversos centros de asistencia/enseñanza. De hecho, la formación post residencia que, desde hace tiempo, se realiza en nuestro país tiene un alto grado de desarrollo en algunas disciplinas, como la carrera de especialización de la Asociación de Mano. También existen muchos programas formadores de las Sociedades o de los distintos centros en cirugía espinal y en los de reconstrucción del adulto (cadera y rodilla). Ortopedia Infantil también tiene un programa de formación básico en algunos centros pediátricos. Sin embargo, en ciertas situaciones, se han observado incertidumbres con respecto a su denominación, el tiempo de duración, los programas y el reconocimiento.

La denominación es todavía incierta en nuestro país. La palabra fellow es un anglicismo, pero se ha aceptado de hecho. La otra opción es becario, aunque tiene, por definición, una connotación distinta. De todas maneras, es importante determinar una definitiva denominación para poder clasificar y distinguir entre pasantías, becarios, fellows. Porque todos ellos tendrán una formación y un reconocimiento distintos fundamentalmente para las eventuales certificaciones.

La duración, íntimamente ligada a la denominación, es importante para el desarrollo de un marco de enseñanza. No es lo mismo una pasantía de semanas sin actividad que una concurrencia elaborada durante meses o años. Aunque estos son extremos difíciles de confundir; no obstante, existen varias situaciones intermedias que pueden no ser claras. El Servicio de Patología Espinal del Hospital Garrahan tiene becarios, que acceden por concurso de oposición y permanecen durante 1 o 2 años cumpliendo funciones activas y a tiempo completo, dentro de un marco de enseñanza. Lo mismo puede observarse en otros Centros asistenciales públicos o privados. En los EE.UU., la mayoría de los fellowships ortopédicos tienen una duración de un año. ${ }^{3}$

Los programas son distintos en sus contenidos y quizás en la duración, pero no en los objetivos y en la dinámica de la educación, así como en las responsabilidades y en la adecuación del trabajo. Encontramos la necesidad de consensuar en los aspectos comunes de especialidades diversas. ${ }^{4}$ En los EE.UU., las subespecialidades utilizan uno de los siguientes tres sistemas que integran también residencias: San Francisco Matching Program, National Resident Matching Program y American Shoulder and Elbow Surgeons Fellowship Matching Program. ${ }^{2,5}$ Un número importante de profesionales completan dos programas usualmente relacionados: deformidad espinal y cirugía mínimamente invasiva, y deporte y hombro/codo. En 2017, las posiciones ofrecidas en los EE.UU. fueron: Columna 123, Reconstrucción en el Adulto 156, Pie y Tobillo 74, Deporte 226, Trauma 84, Pediatría 75, Mano 176, Hombro y Codo 44. 
La misión fundamental de la educación médica en los graduados es formar a esos jóvenes para transformarse en futuros profesionales y académicos. El entrenamiento en cirugía ortopédica se dirige principal e inicialmente hacia la educación global del residente. El entrenamiento de la subespecialidad en el fellowship es también importante y necesario, aunque más específico. ${ }^{6}$ Es verdad que podrían surgir conflictos de límites y posibilidades de enseñanza entre unos y otros. Podría suceder que las actividades necesarias para la formación práctica tuvieran que ser compartidas. Sin embargo, Allen menciona que el proceso educativo que ocurre durante el fellowship puede tener un efecto beneficioso en el proceso de aprendizaje de los residentes, y viceversa. ${ }^{7}$

En resumen, el objetivo del trabajo es coordinar los programas de entrenamiento en las diferentes especialidades de la ortopedia básica. La Asociación Argentina de Ortopedia y Traumatología como entidad madre de todas las especialidades ortopédicas ha encontrado necesario impulsar y desarrollar este importante paso. Estos estudios podrán ayudar tanto a los Directores de Programas como a los profesionales en formación. Entendemos que el camino está abierto para proseguir con su implementación y objetivos, como el análisis y la definición de vacantes ofrecidas y cubiertas, lo que redundará en el conocimiento de las tendencias asistenciales en nuestra materia.

ORCID iD de S. Vedoya: https://orcid.org/0000-0001-5480-5849 ORCID iD de C. Autorino: https://orcid.org/0000-0001-6410-3816 ORCID iD de R. Remondino: https://orcid.org/0000-0002-8388-298X ORCID iD de J. Varaona: https://orcid.org/0000-0003-3540-4809 ORCID iD de C. Pelaez: https://orcid.org/0000-0002-6267-4786 ORCID iD de J. Romagnoli: https://orcid.org/0000-0002-2521-1563 ORCID iD de R. Valentini: https://orcid.org/0000-0002-0330-6721 ORCID iD de J. P. Torrano: https://orcid.org/0000-0002-3941-9090

\author{
ORCID iD de F. Sartor: https://orcid.org/0000-0001-6061-2445 \\ ORCID iD de F. Catapano: https://orcid.org/0000-0001-9190-1928 \\ ORCID ID de M. Parisi: https://orcid.org/0000-0002-5855-7747 \\ ORCID iD de A. Fazio: https://orcid.org/0000-0002-3807-0966 \\ ORCID iD de S. Reciniello: https://orcid.org/0000-0002-0732-4484 \\ ORCID iD de C. Sancineto: https://orcid.org/0000-0002-5190-4312 \\ ORCID iD de F. Reboiras: https://orcid.org/0000-0002-9546-8057 \\ ORCID iD de C. Facioni: https://orcid.org/0000-0003-3502-2093
}

\section{BIBLIOGRAFÍA}

1. Cannada L, Luhmann S, Hu S, Quinn R. The fellowship match process: the history and a report of the current experience. J Bone Joint Surg Am 2015;97(1):e3. https://doi.org/10.2106/JBJS.M.01251

2. Ruddell J, Eltorai A, Depasse J, Kuris E, Gil J, Cho D, et al. Trends in the Orthopaedic Surgery Subspecialty Fellowship Match: Assessment of 2010 to 2017 Applicant and Program Data. J Bone Joint Surg Am 2018;100(21): e139. https://doi.org/10.2106/JBJS.18.00323

3. Daniels A, Mcdonnell M, Born C, Hayda R, Ehrlich M, Trafton P, et al. Critical analysis of a trauma fellowshipmodeled, six-year Orthopaedic Surgery Training Program. J Bone Joint Surg Am 2013;95(15):e108. https://doi. org/10.2106/JBJS.L.00988

4. Harner C, Ranawat A, Niederle M, Roth A, Stern P, Hurwitz S, et al. AOA Symposium. Current state of fellowship hiring: Is a universal match necessary? Is it possible? J Bone Joint Surg Am 2008;90(6):1375-84. https://doi. org/10.2106/JBJS.G.01582

5. Daniels AH, DiGiovanni CW. Is subspecialty fellowship training emerging as a necessary component of contemporary orthopaedic surgery education? J Grad Med Educ 2014;6(2):218-21. https://doi.org/10.4300/ JGME-D-14-00120.1

6. Emery S, Guss D, Kuremsky M, Hamlin B, Herndon J, Rubash H. Resident education versus fellowship trainingconflict or synergy? J Bone Joint Surg Am 2012;94(21):e159. https://doi.org/10.2106/JBJS.K.01331

7. Allen WC. The relationship between residency programs and fellowships in the educational setting. Clin Orthop Relat Res 1990;(257):57-60. PMID: 2379374 
ANEXO

\section{Asociación Argentina de Ortopedia y Traumatología}

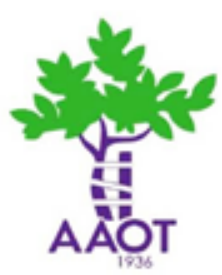

Criterios mínimos y recomendaciones

para la formación post residencia en Ortopedia y Traumatología

\section{Consideraciones generales}

Es cada vez más frecuente que los médicos que han finalizado la residencia opten por continuar su formación en áreas disciplinares específicas de la Ortopedia y Traumatología. Esta formación requiere también de una experiencia educativa en servicio, que se desarrolle en unidades asistenciales de Ortopedia y Traumatologia. Actualmente existen escasos acuerdos con respecto a las condiciones mínimas de esta etapa y una amplia disparidad en cuanto a las ofertas formativas.

La Mesa de trabajo convocada conjuntamente por la Comisión Directiva y el Comité de Residencias y Sistemas Equivalentes de la Asociación Argentina de Ortopedia y Traumatologia invitó a referentes de asociaciones y sociedades integrantes con el fin de intercambiar sus diferentes perspectivas y trayectorias. Se propuso generar un espacio de trabajo que permitiera consensuar las condiciones mínimas de formación a los fines de promover la calidad de las ofertas formativas post residencia y asegurar así una experiencia educativa equivalente independientemente de las unidades asistenciales donde se desarrollen.

Este documento se propone brindar criterios minimos y realizar recomendaciones con respecto a los siguientes ejes:
A. Denominación
B. Dedicación
C. Admisión
D. Programa de formación
E. Condiciones de la sede formadora
F. Actividades formativas - Asistenciales, no asistenciales y de investigación
G. Equipo docente
H. Evaluación y supervisión
I. Condiciones minimas de contratación 


\section{Asociación Argentina de Ortopedia y Traumatología}

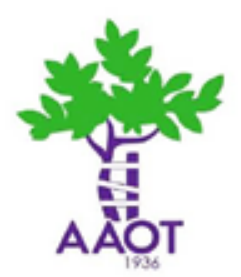

\section{A. Denominación}

La denominación del tipo de formación resulta central para organizar la propuesta formativa, analizar equivalencias y brindar certificaciones y/o titulaciones. Se recomienda ir unificando la oferta hacia una denominación de formación post residencia. Actualmente este tramo formativo es denominado frecuentemente como Beca de Perfeccionamiento o fellowship.

\section{B. Dedicación}

La duración se expresará en meses, y la carga horaria en cantidad de horas de dedicación semanal.

Se recomienda una dedicación semi-exclusiva de 12 meses de duración mínima y una carga horaria mínima estimada en $\mathbf{3 0}$ horas semanales en la se desarrolle la actividad educativa asistencial y educativa no asistencial-académica. Existe la posibilidad de ofrecer formaciones de menor o mayor duración, si se elabora un programa educativo que lo fundamente.

\section{Admisión}

El centro formador deberá establecer claramente sus criterios y mecanismos de admisión. Será requisito para acceder a una formación post residencia:

- Residencia completa realizada en un centro formador acreditado por la AAOT en Ortopedia y Traumatologia adultos o pediátrica o certificado de especialista otorgado por la AAOT

- Haber completado el curso bianual de la AAOT

Las distintas Sociedades integrantes podrán estipular requisitos adicionales.

Se recomienda que la formación post residencial se adecue al calendario formativo de las residencias (junio a mayo).

\section{Programa de formación}

El programa es una herramienta clave para la gestión educativa y es el documento que explicita la propuesta formativa a los postulantes.

Es necesario definir una estructura común básica para todos los programas y asegurar la periódica actualización de los mismos desde lo disciplinar y lo educativo.

Los programas docentes incluirán información con respecto a:

- Fundamentación del programa

- Perfil del egresado

- Objetivos de aprendizaje

- Responsable/s docentes/s 


\section{Asociación Argentina de Ortopedia y Traumatología}

- Contenidos mínimos

- Actividades de aprendizaje (asistenciales y no asistenciales)

- Modalidad de evaluación y supervisión

Nota: A los fines de dar osistencia a lo elaboroción de los programas se propone, a futuro, formular un Instructivo para los responsables docentes.

\section{E. Condiciones de la sede formadora}

Se recomienda que el programa de formación post residencia se desarrolle en unidades asistenciales (UA) acreditadas por la AAOT, y las Sociedades Integrantes. En el caso de no contar con la acreditación, y a los fines de garantizar la calidad de la formación ofrecida, es necesario tener en cuenta las siguientes características de las UA:

- Existen otros programas de residencias, concurrencias o post residencias

- Se reciben alumnos de grado o profesionales de otras UA en calidad de rotantes

- Los profesionales en formación se integran al equipo de salud conformado por los profesionales $y / o$ especialidades que demanda la formación en el área disciplinar correspondiente

- Existe la cantidad y variedad de motivos de consulta y/o pacientes suficientes para formar profesionales en el área disciplinar correspondiente

- Se cuenta con acceso a servicios de diagnóstico (imágenes, anatomia patológica, analítica clínica) e interconsulta

- Dispone de Biblioteca fisica o electrónica y acceso a bases de datos sin costo para los profesionales en formación

- Existen espacios fisicos adecuados para desarrollar actividades formativas no asistenciales (ver punto F)

\section{F. Actividades formativas - Asistenciales, no asistenciales y de investigación}

En relación a las actividades que se desarrollan durante la formación es necesario asegurar las siguientes:

F.1-Actividades formativas asistenciales: se refieren a las prácticas centrales del área disciplinar. Sólo a título de ejemplo: atención de pacientes en consultorio, internación y emergencias; participación en procedimientos diagnósticos quirúrgicos $y / o$ postquirúrgicos;realización de interconsultas u otras. Cada programa formativo podrá definir además las prácticas especificas y relevantes propias de su área disciplinar, así como el número minimo de prácticas indispensables para la formación, en caso de considerarlo de interés.

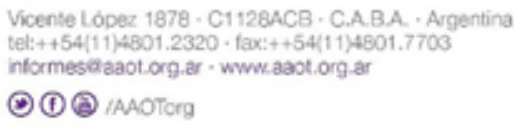




\section{Asociación Argentina de Ortopedia y Traumatología}

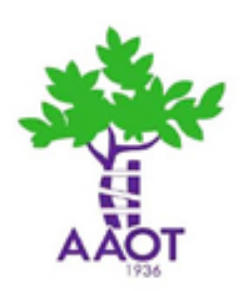

F.2-Actividades formativas no asistenciales: es indispensable que el programa ofrezca a los profesionales en formación la posibilidad de participar de espacios informales de intercambio y discusión sobre la práctica cotidiana, ya que es un componente importante para alcanzar la experticia profesional. A su vez, los programas garantizarán un plan académico minimo que incluya actividades tales como: ateneos; pases de sala; clases; discusión de casos; prácticas de simulación; revisión sistemática de historias clínicas; búsquedas bibliográficas guiadas; participación en congresos o seminarios relevantes del área disciplinar $u$ otras.

F.3-Actividades de investigación: se recomienda que el programa ofrezca la oportunidad de participar en actividades de investigación con niveles crecientes de responsabilidad hacia el interior de una investigación a lo largo de su formación. Se espera que tengan la oportunidad de participar en distintos momentos, fases, y tareas de investigación: por ej. Búsqueda bibliográfica; recolección, procesamiento y análisis de datos; comunicación de resultados.

A modo de orientación, se recomienda la siguiente distribución de tiempo semanal estimado por tipo de actividad:

70\% Actividad asistencial

$25 \%$ Actividad no asistencial - académica

$5 \%$ Actividades de investigación

\section{G. Equipo docente}

La formación en servicio es de tipo incidental, en la que los equipos docentes deben estar capacitados para sacar provecho del potencial educativo de la práctica diaria para la formación de profesionales nóveles. Para ello es necesario formar a los docentes para que sean capaces de planificar ese proceso de enseñanza-aprendizaje en la práctica, con las particularidades de cada UA.

Como se menciona en el punto $D$, el programa especificará el/los responsables docentes de la formación y supervisión de los aprendizajes de los profesionales en formación.

En relación al responsable máximo del programa se espera que:

- cuente con certificación y/o reconocimiento equivalente en el área disciplinar

- sea miembro de la AAOT y/o su asociación/sociedad integrante

- garantice la coordinación y supervisión del cumplimiento del programa 


\section{Asociación Argentina de Ortopedia y Traumatología}

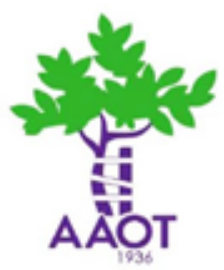

Los entes acreditadores de la formación en ciencias de la salud estiman que el director/responsable docente (o en quién él delegue) debe cumplimentar una formación docente minima de 50 hs reloj de formación en docencia.

\section{H. Evaluación y supervisión \\ La evaluación del desempeño profesional requiere de un seguimiento continuo de los aprendizajes, tanto en relación a conocimientos teóricos como a competencias quirúrgicas. A los fines de realizar un adecuado seguimiento del proceso de formación y de sus resultados se establece la necesidad de:}

H.1- Explicitar en el programa docente la modalidad de evaluación y supervisión que defina responsables, momentos e instrumentos de evaluación a utilizar para tal fin.

H.2- Contar con al menos un instrumento estandarizado de evaluación global del desempeño profesional. La evaluación de la práctica quirúrgica puede realizarse mediante Instrumentos de cotejo quirúrgico.

H.3- Realizar una instancia formal de devolución de esa evaluación, definiendo de manera conjunta progresos, dificultades y orientaciones sobre posibles actividades remediales en caso de ser necesario.

H.4- Se incluirá al menos una instancia de evaluación al promediar la formación y una instancia final de evaluación integradora

Es importante que los evaluadores estimulen un clima de aprendizaje que estimule el intercambio, la reflexión y la activa participación de los profesionales en formación.

\section{Condiciones minimas de contratación}

Dado que la formación requiere de una amplia carga horaria y que el profesional está tramitando o tiene el certificado de especialista y estaría transitando la segunda etapa formativa, se recomienda que cuente con una remuneración mensual que sea definida y comunicada de forma clara y precisa previo al inicio del programa ya que esto contribuye a la calidad de la contratación.

A su vez, los profesionales en formación deberán contar con cobertura médica, seguro de Mala praxis y Aseguradora de riesgo de trabajo (ART) al momento de iniciar su formación. 


\section{Asociación Argentina de Ortopedia y Traumatología}

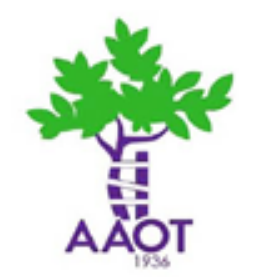

Integrantes de la mesa de trabajo que participaron en la elaboración de este documento:

- Asociación Argentina de Artroscopia - Dr. Federico Spikerman

- Asociación Argentina de Cirugia de la Mano y Reconstructiva del Miembro Superior Dr. Francisco López Bustos

- Sociedad Argentina de Ortopedia y Traumatología Infantil - Dr. Jorge Sueiro

- Sociedad Argentina de Medicina y Cirugia de Pierna y Pie - Dr. Héctor Masaragian y Dr. Luciano Mizdraji

- Asociación Argentina de Traumatología del Deporte - Dr. Mariano Carlos D' elía y Dr. Carlos Yacuzzi

- Asociación Argentina para el Estudio de la Cadera y la Rodilla - Dr. Lisandro Carbó

- Sociedad Argentina de Neuroortopedia - Dr. Dardo Sincosky

- Sociedad Argentina de Patología de la Columna Vertebral - Dr. Luis Marcelo Melo

- Asociación Argentina del Trauma Ortopédico - Dr. Guillermo Gonzalez Naya

- Asociación Argentina para el Estudio de la Reconstrucción y Elongación Osea - Dr. Andrés Greco

- Asociación Argentina de Ortopedia y Traumatología - Dr. Carlos Federico Sancineto.Presidente MC 04/12/2018

- Comité de Residencias y Sistemas Equivalentes - Dres. Carlos Tello, Santiago Vedoya, Carlos Maria Autorino y Rodrigo Remondino

- Asesoras pedagógicas AAOT - Lic. Fabiana Reboiras y Lic. Clara Facioni

- Asistentes administrativas: Tec. Natalia Morgani y Prof. Maria Laura Beltramino

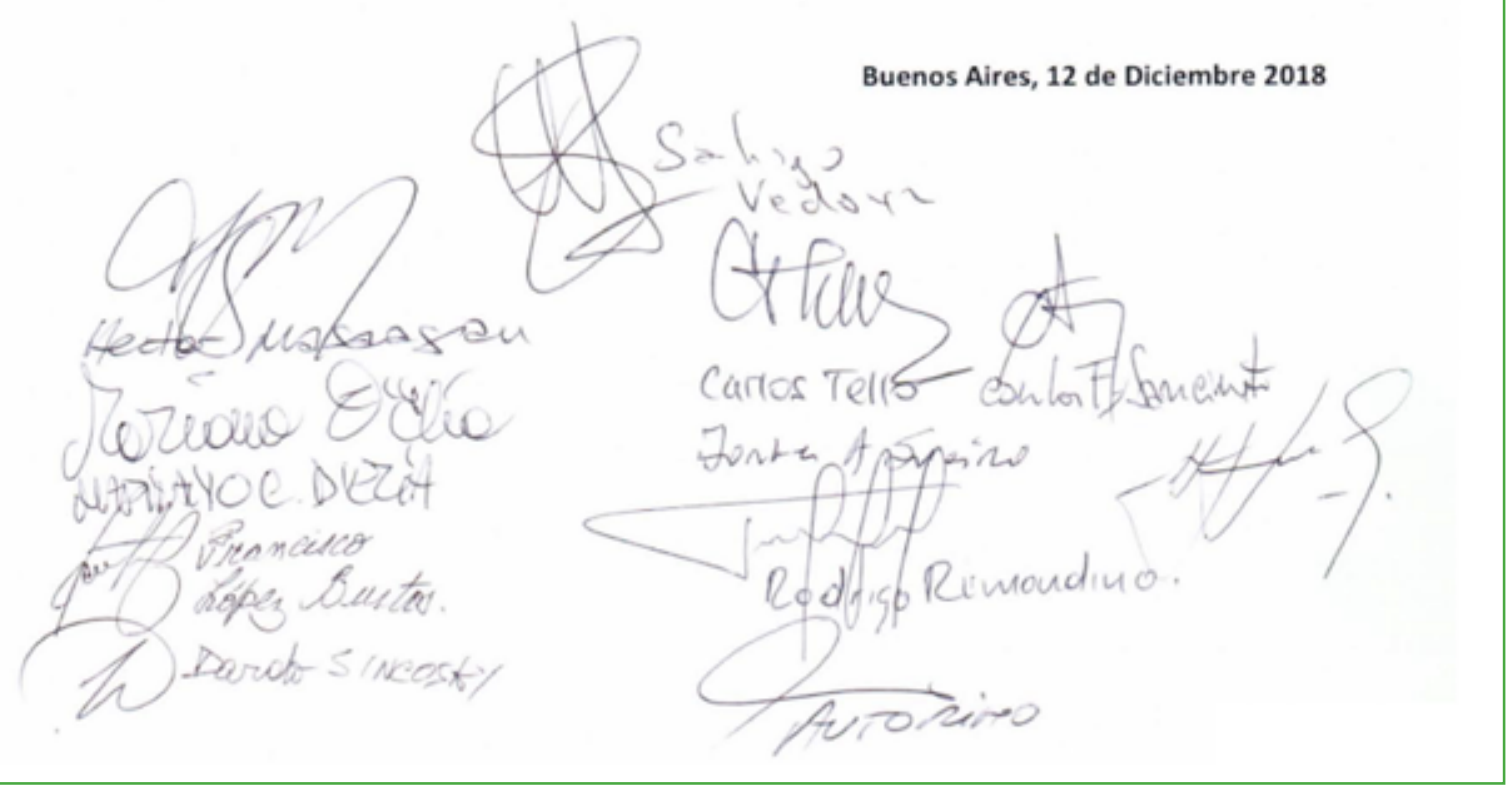


Asociación Argentina de Ortopedia y Traumatología
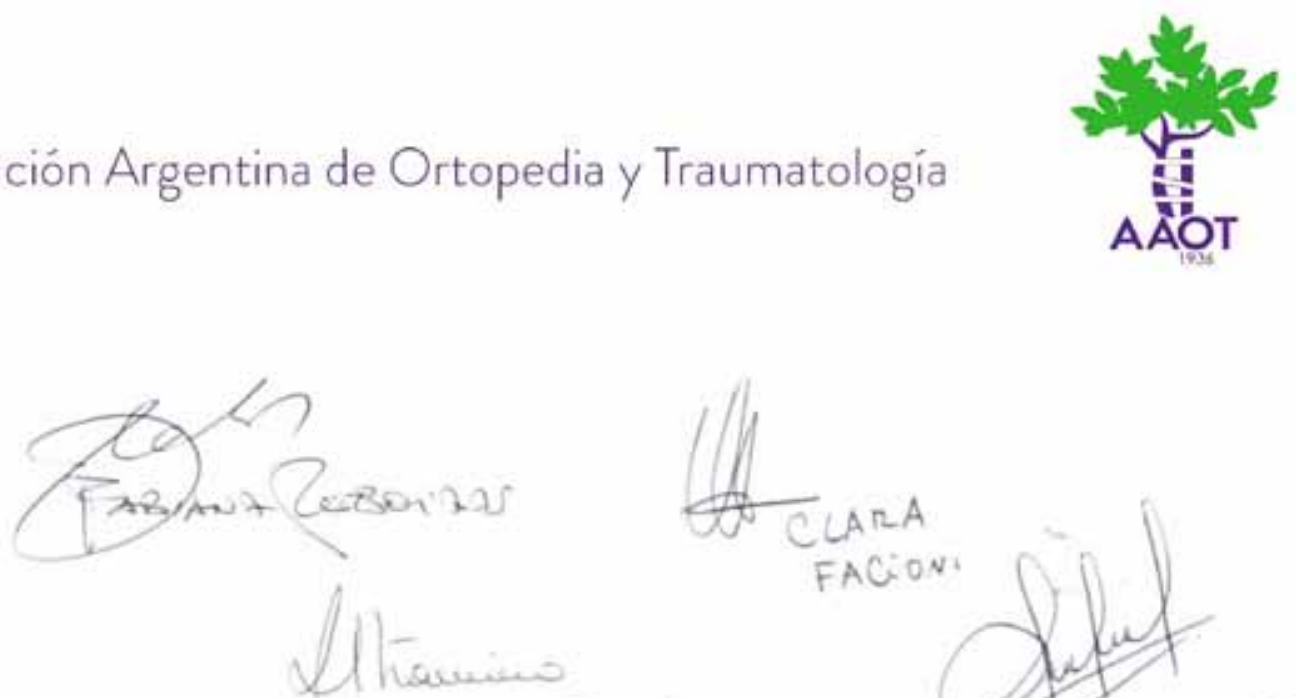

FACION

Manic Bathomino
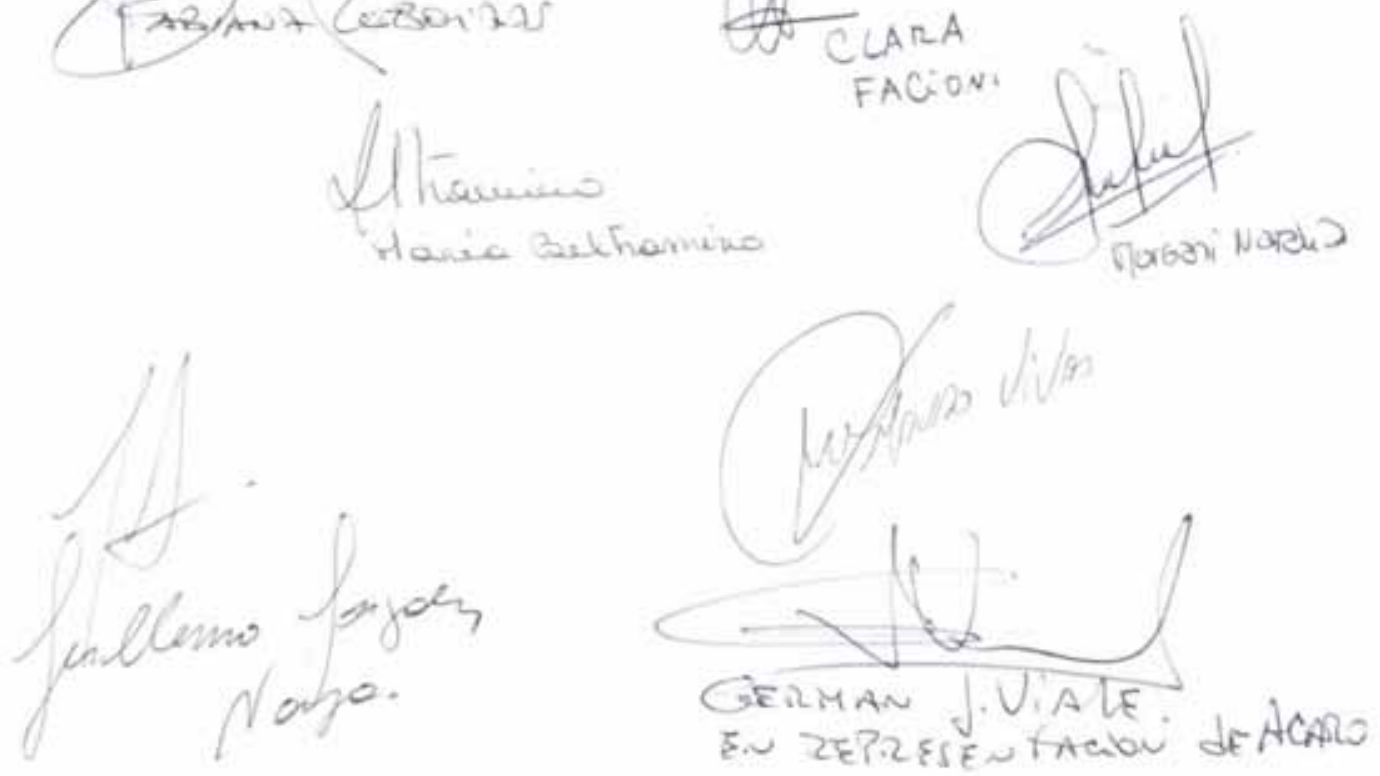